\title{
Percepción del turismo como fUente de bienestar en COMUnidades RURALes de Colima
}

\author{
Carlos Amaya Molinar \\ Irma Magaña Carrillo \\ ILEANA OCHOA LLAMAS \\ Yadira Guerra Montes \\ Facultad de Turismo, Universidad de Colima
}

\begin{abstract}
El turismo basado en la comunidad (TBC) busca el empoderamiento de la población para superar la pobreza y mejorar su calidad de vida, así como lograr beneficios socioculturales y medioambientales. El objetivo del estudio fue explorar la influencia de unidades de negocios turísticos rurales en la superación de la pobreza y en el bienestar de la población, para lo cual se realizó una investigación descriptiva en comunidades rurales de Colima, con técnicas de investigación cualitativas y cuantitativas. Los hallazgos presentados son preliminares y no respaldan los planteamientos del TBC, aunque sí una percepción de la población en torno al turismo como fuente de bienestar.
\end{abstract}

Palabras clave: Turismo, comunidad, pobreza, sostenibilidad, bienestar.

\section{Perception of tourism as a source of Well-being in RURAL COMMUNities OF Colima}

Community-based tourism seeks the population's empowerment so they can overcome poverty, improve their quality of life, and obtain socio-cultural and environmental benefits. The aim of this study was to explore the influence of rural tourist business units in overcoming poverty and improving the well-being of the population. To accomplish this, a descriptive research was conducted in rural communities of Colima using qualitative and quantitative research techniques. The results presented here are preliminary and do not support the approaches of community-based tourism, although there is a perception of tourism as a source of well-being for the population.

Key words: Tourism, community, poverty, sustainability, welfare.

Fecha de recepción: 18 de enero de 2019. Fecha de aceptación: 29 de marzo de 2019

CÓMO CITAR: Amaya, C., Magaña, l., Ochoa, I. y Guerra, Y. (2019). Percepción del turismo como fuente de bienestar en comunidades rurales de Colima. Dimensiones Turísticas, 3(4), 63-86. https://doi.org/10.47557/TKQS1346 
I soporte del trabajo teórico se apoya básicamente en dos campos temáticos que se han desarrollado en el pasado reciente en relación con el estudio de la actividad turística: el turismo basado en la comunidad y el turismo y la superación de la pobreza. La literatura académica atribuye una gran cantidad de virtudes económicas, sociales y medioambientales a la modalidad de operación turística denominada turismo basado en la comunidad ( $\mathrm{TBC}$ ). En el pasado reciente se ha producido una gran cantidad de trabajos académicos en este campo, entre los que destacan los de Asker, Boronyak, Carrard y Paddon (2010), Fernández (2011), Goodwin y Santilli (2009), Hiwasaki (2006), Silva y Wimalaratana (2013), y Tasci, Semrad y Yilmaz (2013), entre otros.

En el ámbito institucional, han surgido iniciativas por parte de organismos internacionales como el Banco Mundial y la Organización Mundial del Turismo para impulsar el turismo como una herramienta con la cual superar la pobreza, respaldados por un conjunto de trabajos académicos de autores como Croes y Rivera (2015), Donaldson (2007), y Jamieson, Goodwin y Edmunds (2004); el Gobierno de la República (2013) reconoce este potencial del turismo para sacar a la población de la pobreza en su Plan Nacional de Desarrollo, mientras que el estudio bibliométrico de Gutiérrez-Pérez, Medina-Muñoz y Medina-Muñoz (2014) documenta su relevancia académica actual.

En el trabajo de campo sobre твс se examinan cuestiones como la sostenibilidad, en sus vertientes económica, social y ambiental, así como algunos de los factores de éxito y beneficios que se le atribuyen. En el tema del turismo y superación de la pobreza, básicamente se explora la percepción del turismo como fuente de bienestar en las comunidades rurales con unidades de negocios turísticos investigadas.

Además de la revisión de la literatura académica sobre esos temas, se realizó trabajo de campo en seis comunidades rurales, obteniendo 908 cuestionarios de la población para conocer su percepción del turismo como fuente de bienestar; por otra parte, se efectuaron entrevistas a líderes de las unidades de negocios y a más de 40 trabajadores para explorar asuntos relacionados con la gestión de los negocios turísticos. De la misma manera, se hicieron observaciones de la operación turística, concentrándose en temas vinculados con el aspecto de la unidad de negocios, la sustentabilidad de la operación y la afluencia de visitantes.

Al inicio, la investigación se apoyó principalmente en el campo teórico del TBC para aproximarse al estudio de las unidades de negocios incluidas en la muestra; sin embargo, los primeros resultados llevaron a buscar otro tipo de soportes académicos que permitieran comprender los fenómenos observados en los diversos objetos de estudio abordados. A pesar de la proximidad geográfica, entre los principales resultados arrojados por el trabajo de campo se encuentran el carácter único y original de cada una de las unidades de negocios estudiadas, con sus formas de organización, sus problemas específicos y sus resultados. Algunas unidades de negocios presentan situaciones de estancamiento permanente, una más prácticamente desapareció por problemas organizacionales, otra manifiesta problemas de sustentabilidad ambiental y solo un par destaca por sus logros económicos positivos y los empleos que genera.

Es oportuno señalar que en este documento se ofrecen resultados preliminares, con una visión panorámica. La información recabada se encuentra aún en proceso de análisis y 
posteriormente se publicarán resultados en profundidad por cada unidad de negocios; de igual modo, los avances alcanzados en los reportes exigen regresar a recopilar información complementaria.

\section{Antecedentes}

En este apartado se presenta información sobre los campos de estudio básicos que fundamentan la investigación. En primer lugar se expone información acerca del modelo teórico denominado turismo basado en la comunidad ( $\mathrm{TBC}$ ), en un momento previo a la exposición de los planteamientos sobre el turismo como herramienta para la superación de la pobreza y la generación de bienestar entre grupos de población excluidos de los beneficios económicos. Los temas se presentan en ese orden asumiendo, en el plano de la teoría, que el TBC funciona como un instrumento para superar la pobreza en la muestra estudiada.

\subsection{El turismo basado en la comunidad en la literatura académica}

En las últimas décadas se han realizado en diversos países del mundo numerosos trabajos sobre el TBC, los cuales han analizado estudios de casos, al igual que desarrollos de modelos teóricos y conceptuales. En los trabajos han participado tanto académicos como organismos internacionales y organizaciones no gubernamentales. Una definición típica del TBC incluye ambientes culturales no occidentales, donde aspectos como el estilo de vida, el folclore, la cultura, la vestimenta, la comida, la bebida y los ambientes naturales forman parte de los atractivos turísticos de las unidades de negocios. Con frecuencia, las comunidades se ubican en zonas rurales alejadas de los núcleos urbanos y, comúnmente, marginadas (Tasci, Semrad y Yilmaz, 2013).

Entre los atributos del TBC se menciona la sostenibilidad, sugiriendo que las operaciones de atención a los visitantes deben realizarse atendiendo a criterios de protección del medio ambiente, a resultados financieros positivos con rentabilidad económica y a una apropiada distribución social de beneficios; esto es, se propone una sostenibilidad en sus vertientes ambiental, económica y social. Otra característica de esta modalidad turística se refiere a la participación directa de la comunidad en la gestión de las operaciones turísticas, involucrando a la mayor proporción posible de integrantes de la comunidad, si no es posible en la operación, sí en la distribución de beneficios.

Desde el punto de vista económico, se menciona que uno de los principales propósitos de la gestión turística comunitaria es contribuir con beneficios económicos y disminuir la pobreza de la población. En la vertiente sociocultural, un rasgo atribuido a este modelo de operación turística es la participación de los visitantes en las manifestaciones culturales, lúdicas y productivas de la comunidad; otras definiciones señalan como característica de esta modalidad la pernocta en hospedajes locales.

En un contexto más amplio, el TBC se inscribe en el campo de las empresas de base comunitaria, que busca movilizar a los miembros de una comunidad para identificar sus 
propios problemas y necesidades, tomar decisiones conjuntas y asumir responsabilidades (The World Bank, 2013). Más puntualmente, Fernández (2011) define a la empresa de base comunitaria como un emprendimiento social colectivo de un grupo de personas que comparte valores, identidad y capital social, procediendo a crear y gestionar una empresa con miras a producir un valor social que le permita obtener beneficios sociales, culturales y financieros.

Una cuestión referida con frecuencia en la literatura académica sobre las empresas de base comunitaria en general y sobre el TBC en particular es el empoderamiento de la colectividad, que lleva a sus integrantes a movilizarse para adquirir un papel más activo en la toma de decisiones, controlar las actividades que influyen en su vida y gestionar sus recursos. El propósito del empoderamiento es que la población de la comunidad supere su estado de subordinación y marginación socioeconómica (Goodwin y Santilli, 2009).

Como en cualquier destino turístico, el atractivo constituye el motivo fundamental que lleva a los visitantes a desplazarse para consumirlo. En el TBC pueden identificarse numerosas categorías de atractivos turísticos, entre los que se mencionan los naturales, culturales, paisajes, recorridos y actividades productivas. En general, los atractivos de las empresas de TBC suelen ser muy similares a los atractivos turísticos comunes; la diferencia radica en la forma de operar los servicios turísticos, misma situación que se presenta cuando se abordan temas de gestión de la organización, como la participación en la toma de decisiones, la mercadotecnia, la rendición de cuentas, etcétera.

En la literatura académica se han postulado algunos principios para la gestión de proyectos y unidades de negocios de TBC; algunos de ellos se señalan en la misma definición del término, como la participación y el control de las actividades turísticas por la comunidad, la planeación participativa y la equidad en la distribución de los beneficios. Otros lineamientos propuestos son la adaptación del sistema económico de la unidad de negocios turística a las características locales, la respuesta a los intereses y necesidades de la comunidad, la complementariedad de la operación turística con sus actividades tradicionales y, un tema trascendental, la implementación de un sistema de formación para el desarrollo de las competencias operativas necesarias para la apropiada gestión del negocio turístico (Asker, Boronyak, Carrard y Paddon, 2010).

La literatura académica atribuye al TBC un conjunto de beneficios económicos, socioculturales y ambientales que se presentan en mayor o menor grado en las localidades donde opera; en el cuadro 1 se enlistan algunos de estos beneficios coincidentes con los planteamientos sobre sustentabilidad. Entre ellos, algunos de los más tangibles son los económicos, ya que los habitantes de las comunidades pueden percibirlos directamente. Los beneficios socioculturales resultan menos tangibles, su maduración es paulatina y de largo plazo; entre estos se encuentran la mejora del capital social, el fortalecimiento de la identidad local, el desarrollo del capital humano, la mejora en la calidad de vida y el desarrollo comunitario. Los beneficios ambientales conferidos a esta modalidad turística resultan cruciales, frecuentemente el éxito de la empresa depende de la conservación de 
Cuadro 1. Algunos beneficios del turismo basado en la comunidad

\begin{tabular}{|c|c|c|}
\hline Económicos & SOCIOCULTURALES & AMBIENTALES \\
\hline $\begin{array}{l}\text { Incremento de empleos e } \\
\text { ingresos }\end{array}$ & $\begin{array}{l}\text { Desarrollo de capital humano } \\
\text { mediante educación y } \\
\text { capacitación }\end{array}$ & $\begin{array}{l}\text { Uso sustentable y conservación } \\
\text { del capital natural de la } \\
\text { comunidad }\end{array}$ \\
\hline Superación de la pobreza & $\begin{array}{l}\text { Mejora del capital social y de } \\
\text { las relaciones con organismos } \\
\text { externos }\end{array}$ & $\begin{array}{l}\text { Diversificación del uso de } \\
\text { recursos }\end{array}$ \\
\hline Recuperación económica & $\begin{array}{l}\text { Preservación del patrimonio } \\
\text { cultural e histórico de la } \\
\text { comunidad }\end{array}$ & $\begin{array}{l}\text { Uso no consuntivo de los } \\
\text { recursos naturales }\end{array}$ \\
\hline Desarrollo de infraestructura & $\begin{array}{l}\text { Mejora de la calidad de vida } \\
\text { y de la infraestructura de la } \\
\text { comunidad }\end{array}$ & $\begin{array}{l}\text { Incremento de la conciencia } \\
\text { ambiental }\end{array}$ \\
\hline Diversificación económica & $\begin{array}{l}\text { Desarrollo comunitario, mejora } \\
\text { de la identidad comunitaria, } \\
\text { sensación de orgullo, cohesión } \\
\text { social y empoderamiento }\end{array}$ & $\begin{array}{l}\text { Mayor comprensión de la } \\
\text { relación entre el medio ambiente } \\
\text { y el desarrollo económico } \\
\text { sustentable }\end{array}$ \\
\hline
\end{tabular}

Fuente: Twining-Ward (2007).

los recursos naturales: un atractivo natural exitoso puede resultar víctima de su propio éxito, pues corre el riesgo de degradarse por sobreexplotación (Twining-Ward, 2007).

De la misma manera, la literatura académica postula un conjunto de principios para la organización y operación de las unidades de negocios de TBC, que pueden contribuir a su éxito comercial y socioeconómico (cuadro 2). Algunos de estos principios enunciados son muy similares a los beneficios y a las definiciones de la modalidad turística mencionados anteriormente; en este sentido, se enuncian la organización de la comunidad y su empoderamiento; la participación e involucramiento de los agentes interesados en la gestión, operación y toma de decisiones en la unidad de negocios; la participación y distribución equitativa de costos, inversiones, actividades y beneficios entre los integrantes de la comunidad (Asker et al., 2010). Un conjunto de autores menciona la sustentabilidad ambiental, social y cultural como principio organizacional del TBC (Goodwin y Santilli, 2009; Salazar, 2012; Kyrgyz Community Based Tourism Association, 2013).

Asker et al. (2010), Silva y Wimalaratana (2013), Salazar (2012) y el Banco Mundial (The World Bank, 2013) proponen integrar a las unidades de negocios de turismo comunitario en mecanismos de participación público privada (PPP) con instituciones gubernamentales de diversos niveles, organizaciones no gubernamentales y empresas. Estos mismos autores señalan uno de los principios esenciales de cualquier actividad de gestión: la transparencia y la rendición de cuentas en la administración de los recursos de la unidad de negocios frente a la comunidad.

En el ámbito específico de la gestión de negocios se subrayan como importantes principios de operación la rentabilidad y la viabilidad económica de los proyectos, aludiendo 
Cuadro 2. Algunos principios para el éxito del turismo basado en la comunidad mencionados en la literatura académica

\begin{tabular}{|c|c|c|}
\hline Proximidad al mercado turístico & Inclusión de stakeholders & $\begin{array}{l}\text { Gestión por habitantes de la } \\
\text { comunidad }\end{array}$ \\
\hline Ventaja competitiva & $\begin{array}{l}\text { Conservación del medio } \\
\text { ambiente }\end{array}$ & Conservación del capital social \\
\hline Motivación de la comunidad & Educación, capacitación & $\begin{array}{l}\text { Transparencia y rendición de } \\
\text { cuentas }\end{array}$ \\
\hline Calidad del producto & Viabilidad comercial & Orientación a la demanda \\
\hline Gestión financiera & $\begin{array}{l}\text { Distribución equitativa de } \\
\text { costos y beneficios }\end{array}$ & Existencia de infraestructura básica \\
\hline
\end{tabular}

Fuente: Elaboración propia.

a numerosos casos de proyectos de turismo comunitario que van a la quiebra y son abandonados una vez que se suspenden los apoyos externos o institucionales (Salazar, 2012; Kyrgyz Community Based Tourism Association, 2013; Silva y Wimalaratana, 2013; Goodwin y Santilli, 2009). En este contexto, Dixey (2005) es un autor que enfoca sus planteamientos en la gestión comercial de la empresa, mencionando como principios la calidad del producto, el aprovechamiento de ventajas competitivas, la administración financiera, la asociación con empresas turísticas y la proximidad a los mercados. El Banco Mundial (The World Bank, 2013) designa la orientación a la demanda como principio organizacional. Por su parte, el Thailand Community Based Tourism Institute (2013) y Goodwin y Santilli (2009) destacan como principio la formación de los miembros de la comunidad mediante acciones de educación y capacitación; en tanto, Asker et al. (2010), la disposición de una adecuada infraestructura de acceso y sólidos mecanismos de mercadotecnia.

La literatura académica también registra limitaciones del TBC. Goodwin y Santilli (2009) mencionan información sobre 200 unidades de negocios de TBC que muestran una tasa de ocupación de $5 \%$ para proveedores de hospedaje, señalando que el resultado más común para los proyectos de turismo en esta modalidad es que colapsen una vez que se agota el financiamiento, especialmente por dificultades de acceso al mercado y dirección.

Goodwin y Santilli (2009) y Tasci et al. (2013) enumeran un conjunto de estudios de casos de unidades de negocios de TBC en diversos países, la mayoría de ellos considerados economías emergentes o en vías de desarrollo. Su análisis permitió identificar factores de éxito, de fracaso o de ralentización; cada una de las unidades de negocios estudiadas presenta características únicas. El objetivo central perseguido en todos los casos es llevar a las unidades de negocios comunitarias desde su origen, apoyado por organizaciones externas, hasta la autosuficiencia, la autogestión y la sustentabilidad de la operación turística en sus vertientes económica, sociocultural y ambiental. El trabajo de estos autores 
ha hecho posible determinar las circunstancias que permiten a un proyecto de TBC salir adelante o malograrse.

En esta investigación, en el campo de estudios del TBC, se examinan aspectos relacionados con la forma de organización, empleos generados, sostenibilidad ambiental, problemática específica identificada y afluencia estimada de visitantes.

\subsection{Turismo y superación de la pobreza}

La información recabada mediante la revisión de la literatura académica sobre TBC sugiere que esta modalidad de operación turística resulta apropiada para contribuir a mejorar la calidad de vida e incrementar el ingreso y los niveles de bienestar entre la población de las comunidades que componen la muestra investigada.

El estudio bibliométrico realizado por Gutiérrez-Pérez et al. (2014) ilustra que los trabajos académicos sobre el turismo como herramienta para el alivio de la pobreza publicados en revistas indexadas de diferentes disciplinas han crecido en forma sostenida, pasando de un trabajo en 1999 a 20 en 2011; en ese periodo se identificó un total de 80 artículos publicados, derivados de estudios efectuados en 36 países de los distintos continentes del planeta, comprobando la relevancia que el tema ha adquirido en la comunidad científica. Los trabajos se han publicado en revistas académicas de muy variadas disciplinas, aunque temáticamente se concentran en las áreas de gestión y ciencias sociales. En cuanto a las tipologías de turismo analizadas, los autores señalan que los trabajos tienden a concentrarse en los temas de turismo en general, turismo de naturaleza y turismo comunitario. Las investigaciones revisadas comprenden aproximaciones teóricas y empíricas, y emplean tanto métodos cuantitativos como cualitativos de diversa índole.

La pobreza se ha definido en términos simplistas y complejos. El Banco Mundial (The World Bank, 2018) establece una línea fijada entre 1.90 dólares estadounidenses para definir la pobreza en términos de paridad de poder de compra del año 2011. Datos proporcionados por esta institución manifiestan que la pobreza extrema ha pasado de 35.9 a $11 \%$ entre 1990 y 2015, mostrando una tendencia decreciente, con un comportamiento desigual entre diversas regiones del planeta, pues zonas del África subsahariana y el sur de Asia están excluidas de tal tendencia; de la misma manera, persisten diferencias entre variables como los medios rural y urbano y el género. México aparece en estas estadísticas entre los 40 países con mayor proporción de población en condición de pobreza y mayor desigualdad en la distribución del ingreso.

El Consejo Nacional de Evaluación de la Política de Desarrollo Social (Coneval, 2018) propone un enfoque multidimensional para medir la pobreza, y determina que la población en situación de pobreza es aquella que carece de por lo menos uno de los derechos sociales y de los ingresos requeridos para adquirir los bienes y servicios con los cuales satisfacer sus necesidades, estableciendo la línea de pobreza por ingresos en el valor total de las canastas alimentaria y no alimentaria por persona al mes (Coneval, 2019). 
También el Coneval (2019) informa que desde el inicio de sus mediciones en 2008, hasta 2016, la población mexicana en situación de pobreza pasó de 44.4 a 43.6 \%, registrando una disminución de 0.08 \% en el periodo, prácticamente $0.01 \%$ anual, por lo que resulta difícil mencionar un incremento significativo de la proporción de la población sobre la línea de bienestar económico, con la disminución de las carencias sociales presentando resultados mixtos en las diversas regiones del país. En el caso de Colima, la población en situación de pobreza pasó de 27.4 a 33.6 \% mostrando una tendencia negativa superior a la media nacional.

Entre los grupos sociales, alrededor de $75 \%$ de la población indígena y 60 \% de la población rural presentan niveles de pobreza superiores a la media nacional, al igual que 50 \% de menores de 18 años y personas con algún tipo de discapacidad. Tres de cada cuatro mexicanos sufren por lo menos una carencia social, destacando la falta de acceso a la seguridad social. Chiapas, Guerrero, Chiapas y Veracruz exhiben la mayor proporción de población en situación de pobreza (Coneval, 2019). En este contexto de pobreza mayoritaria entre la población rural nacional, resulta comprensible que los habitantes de las comunidades busquen aprovechar cualquier oportunidad que se les presente de generar ingresos para sus familias, como es la actividad turística.

Durante la reunión Cumbre del Milenio 2000, la Organización de la Naciones Unidas identificó a la pobreza como uno de los mayores desafíos mundiales, y fijó a la erradicación de la pobreza extrema como uno de sus Objetivos de Desarrollo del Milenio. En 2002, la Organización Mundial del Turismo respondió anunciando la Iniciativa ST-EP (Sustainable Tourism-Eliminating Poverty) en la Cumbre Mundial sobre Desarrollo Sostenible de Johannesburgo. Esta iniciativa promueve el alivio de la pobreza a través del apoyo a proyectos de desarrollo turístico sostenible.

Desde fines del siglo xx, la Organización Mundial del Turismo ha propuesto utilizar el turismo como herramienta para superar la pobreza, destacando el programa ST-EP y un conjunto de manuales e investigaciones orientado a ese fin. Señala que en muchos países emergentes el turismo es la opción de desarrollo económico más viable y sostenible y una importante fuente de divisas -el ritmo de crecimiento anual de llegadas en destinos emergentes duplica el de las economías avanzadas-. Asimismo, propone que la disminución de la pobreza debe ser el objetivo central del desarrollo turístico, e identifica varias formas en que el turismo beneficia a la población: empleo directo e indirecto, venta de bienes y servicios, incentivos fiscales, donaciones, construcción de infraestructura y operación de empresas turísticas (World Tourism Organization, 2004 y 2010).

Investigadores de diversos países han abordado el tema desde la academia. Jamieson et al. (2004) destacan que el turismo puede generar impactos positivos o negativos en la población receptora, por lo que deben medirse sus efectos económicos, ambientales y sociales en comunidades rurales y urbanas. Goodwin (2006) indica que se conoce poco sobre el impacto del turismo en la superación de la pobreza: los resultados del turismo se expresan en términos macroeconómicos abstractos y la política turística tiende a orientarse a la captación de divisas. Este autor encontró impactos positivos significativos en casos 
de estudio de trabajadores en situación de pobreza en operaciones turísticas en Egipto, Tanzania y Gambia.

La generalidad de los trabajos consultados sobre el impacto del turismo en la superación de la pobreza le atribuye resultados positivos, aunque puntualiza que tales efectos pueden optimizarse mediante políticas específicas y que no siempre es sencillo cuantificarlos.

Blake, Saba, Sinclair y Kuhl (2008) estudian el impacto del turismo en la economía brasileña, encontrando que todos los grupos sociales de ese país se benefician, pero los pobres en menor medida. Donaldson (2007) y Croes (2014) analizan los efectos mixtos del turismo y los resultados diferenciados en la población en situación de pobreza en China y en América Latina. Hawkins y Mann (2007) cuestionan la decisión del Banco Mundial de abandonar el financiamiento a proyectos turísticos a mediados de la década de 1970, a la luz de los resultados obtenidos, señalando también la necesidad de presentar mayores evidencias de los impactos del turismo en la reducción de la pobreza. Los trabajos de Manyara y Jones (2007), Mitchell y Ashley (2009), Scheyvens y Russell (2012), Torres y Momsen (2004), y Zapata, Hall, Lindo y Vanderschaeghe (2011) coinciden en que el turismo contribuye a disminuir la pobreza, aunque tal contribución podría ser mayor operando con políticas y condiciones específicas.

El Plan Nacional de Desarrollo 2013-2018 de México (Gobierno de la República, 2013) considera al turismo como un sector estratégico con alta capacidad para generar empleo e incrementar los mercados para las pequeñas y medianas empresas y con capacidad para preservar la riqueza natural y cultural de la nación, agregando que 87 \% de la población en municipios turísticos en nuestro país tiene un nivel de marginación "muy bajo”, mientras que ese dato en los municipios no turísticos es de $9 \%$. En línea con los planteamientos de la Organización Mundial del Turismo, establece que todas las políticas de desarrollo del sector deben enfocarse a incrementar la contribución del turismo a la reducción de la pobreza y a la inclusión social.

En sus trabajos sobre el impacto socioeconómico del turismo en el bienestar de la población, Croes (2014) y Croes y Rivera (2015) apuntan que el turismo es un poderoso instrumento para superar la pobreza mediante mecanismos directos e indirectos. A la definición oficial de esta condición basada en una línea de ingreso que imposibilita el acceso a límites mínimos de consumo y al desarrollo de las capacidades, los autores agregan situaciones de disparidad, rechazo, fragilidad, aislamiento, carencias y penurias. Las investigaciones establecen la conveniencia de apoyar a los pobres de manera altruista, pues invertir en su alimentación, formación, servicios médicos e infraestructura impulsaría la productividad de la economía en general.

En el campo de estudios del turismo y superación de la pobreza se emplean los planteamientos de Croes (2014) y Croes y Rivera (2015) en las variables utilizadas para la medición de la percepción del turismo como fuente de bienestar y de satisfacción con la vida entre la población de las comunidades rurales. 


\section{Metodología}

El estado de Colima se ubica en el occidente de México, sobre la costa del Pacífico mexicano. Se caracteriza por su terreno accidentado y por presentar una acentuada pendiente desde la cumbre del Volcán de Fuego hasta la línea costera, con una altitud media sobre el nivel del mar de 570 metros. La temperatura promedio en los municipios de Colima y Comala es de alrededor de $25^{\circ} \mathrm{C}$, con lluvias concentradas entre junio y octubre. Su vegetación es muy diversa, compuesta por selvas bajas, bosques de pino, encino y vegetación de montaña, con alguna presencia de matorrales y pastizales (Instituto Nacional de Estadística y Geografía, 2018). La riqueza de la biodiversidad de su ecosistema, los diversos cuerpos de agua existentes y los rasgos fisiográficos de la región favorecen la práctica de actividades de recreación en contacto con la naturaleza.

Durante el desarrollo de la investigación se aplicaron diversas técnicas, cuantitativas y cualitativas. Se estudiaron seis unidades de negocios ubicadas en comunidades rurales de dos municipios de la zona norte del estado de Colima: Colima y Comala; su operación gira mayormente alrededor de cuerpos de agua y manantiales, aunque algunas son establecimientos de alimentos y bebidas. La investigación de campo consistió en la aplicación de encuestas, entrevistas y guías de observación; estuvo a cargo de profesores y estudiantes de licenciatura y posgrado de la Facultad de Turismo de la Universidad de Colima. El trabajo de campo se realizó entre agosto de 2016 y junio de 2017.

En el campo de las técnicas cuantitativas se aplicó una encuesta que logró reunir 908 cuestionarios, empleando un procedimiento de muestreo de conveniencia; en términos de proporciones, el número de cuestionarios cubre $11 \%$ del total de la población de las comunidades estudiadas, aunque esta proporción no se distribuye equitativamente entre todas ellas, pues en Suchitlán solo se cubrió 8 \% de la población, mientras que en Acatitán, 23 \%. El cuestionario se adaptó del trabajo de Croes y Rivera (2015), buscando detectar la percepción del impacto de la actividad turística en el bienestar de la población y la satisfacción con la vida.

Se aplicaron entrevistas al personal que presta sus servicios en las unidades de negocios examinadas, orientadas a extraer información sobre las características y la percepción de la calidad de los empleos generados por la actividad turística, con lo cual se obtuvo un total de 42 instrumentos; en la comunidad de Zacualpan no se efectuaron entrevistas porque su unidad de negocios no está operando. También se aplicaron entrevistas a los dirigentes de las unidades de negocios, con el propósito de conocer las prácticas de gestión de las unidades. Asimismo se realizó observación estructurada en comunidades y unidades de negocios.

Como ya se comentó, la presente investigación se encuentra actualmente en proceso y la gran cantidad de información recabada está siendo procesada por diversos métodos de análisis estadístico y cualitativo. El cuadro 3 muestra la cantidad de técnicas e instrumentos de investigación aplicados en las comunidades estudiadas. 
Cuadro 3. Métodos y técnicas utilizados en el estudio

\begin{tabular}{|c|c|c|c|c|c|c|}
\hline \multicolumn{7}{|c|}{ Avances en el proceso de investigación } \\
\hline $\begin{array}{l}\text { Unidad de } \\
\text { negocios }\end{array}$ & $\begin{array}{c}\text { Centro } \\
\text { Recreativo } \\
\text { La María }\end{array}$ & $\begin{array}{c}\text { Centro } \\
\text { Turístico } \\
\text { Carrizalillo }\end{array}$ & $\begin{array}{c}\text { Parador } \\
\text { "Gastronómico" }\end{array}$ & $\begin{array}{c}\text { Parador } \\
\text { "Gastronómico" }\end{array}$ & $\begin{array}{l}\text { Manantiales } \\
\text { de Zacualpan }\end{array}$ & El Cahuite \\
\hline Municipio & & & Comala & & & Colima \\
\hline Comunidad & La Becerrera & Suchitlán & $\begin{array}{l}\text { Cofradía de } \\
\text { Suchitlán }\end{array}$ & La Nogalera & Zacualpan & Acatitán \\
\hline $\begin{array}{l}\text { Población de la } \\
\text { comunidad }\end{array}$ & 274 & 4083 & 1601 & 354 & 1724 & 191 \\
\hline $\begin{array}{l}\text { Encuesta a } \\
\text { trabajadores }\end{array}$ & 7 & 4 & ND & 17 & ND & 14 \\
\hline Encuesta (908) & 24 & 326 & 163 & 64 & 288 & 43 \\
\hline \% Población & $9 \%$ & $8 \%$ & $10 \%$ & $18 \%$ & $17 \%$ & $23 \%$ \\
\hline $\begin{array}{l}\text { Entrevistas a } \\
\text { dirigentes }\end{array}$ & Sí & Sí & Sí & En proceso & Sí & Sí \\
\hline Observación & Sí & Sí & Sí & Sí & Sí & Sí \\
\hline
\end{tabular}

Fuente: Elaboración propia.

\section{Resultados}

Cinco de las comunidades investigadas se localizan en el municipio de Comala y una más en Colima, y se advierten importantes diferencias en su población; también puede percibirse que los empleos directos creados en las unidades de negocios son relativamente pocos, y Acatitán y La Nogalera generan una cantidad proporcionalmente mayor. El cuadro 4 exhibe los atractivos y servicios de las unidades de negocios estudiadas. Así, dos unidades de negocios se basan en lagunas, dos en servicios de alimentos y bebidas y dos más son balnearios alimentados por manantiales. Las unidades de negocios basadas en lagunas presentan una mayor cantidad de servicios y una mayor complejidad, seguidas por los balnearios y por los corredores gastronómicos, con una oferta simple de alimentos y bebidas.

La totalidad de las comunidades donde se ubican las unidades de negocios examinadas cuenta con infraestructura de servicios básicos, como carretera pavimentada, electricidad, agua potable, drenaje, cobertura de telefonía fija y celular y televisión abierta; aunque no existen instalaciones en todas las comunidades incluidas, la población tiene acceso a servicios educativos y de salud básicos. 
CuAdro 4. Atractivos y servicios de las unidades de negocios estudiadas

\begin{tabular}{|c|c|c|c|}
\hline $\begin{array}{l}\text { Unidad de } \\
\text { negocios }\end{array}$ & Atractivo & Servicios & Complementos \\
\hline $\begin{array}{l}\text { Centro Recreativo } \\
\text { La María }\end{array}$ & Laguna & $\begin{array}{l}\text { Hospedaje, alimentos y } \\
\text { bebidas, cobertizos, tienda }\end{array}$ & $\begin{array}{l}\text { Alquiler de lanchas, } \\
\text { pesca, albercas, canchas, } \\
\text { campamento }\end{array}$ \\
\hline $\begin{array}{l}\text { Centro Turístico } \\
\text { Carrizalillo }\end{array}$ & Laguna & $\begin{array}{l}\text { Hospedaje, alimentos y } \\
\text { bebidas, cobertizos }\end{array}$ & $\begin{array}{l}\text { Alquiler de lanchas, } \\
\text { bicicletas y caballos, } \\
\text { canchas, juegos infantiles, } \\
\text { gotcha }\end{array}$ \\
\hline $\begin{array}{l}\text { Parador } \\
\text { “Gastronómico" } \\
\text { Cofradía de } \\
\text { Suchitlán }\end{array}$ & Gastronomía & $\begin{array}{l}\text { Alimentos y bebidas: } 4 \\
\text { cocinas económicas, } 2 \\
\text { cafeterías, } 1 \text { merendero }\end{array}$ & \\
\hline $\begin{array}{l}\text { Parador } \\
\text { “Gastronómico" } \\
\text { La Nogalera }\end{array}$ & Gastronomía & Alimentos y bebidas & \\
\hline $\begin{array}{l}\text { Manantiales de } \\
\text { Zacualpan }\end{array}$ & Manantial & $\begin{array}{l}\text { Balneario natural, alimentos y } \\
\text { bebidas, cobertizos }\end{array}$ & \\
\hline El Cahuite & Manantial & $\begin{array}{l}\text { Albercas, toboganes, } \\
\text { cobertizos, tienda }\end{array}$ & Canchas, campamento \\
\hline
\end{tabular}

Fuente: Elaboración propia.

\subsection{Resultados por unidad de negocios}

El cuadro 5 es un resumen de los resultados generales preliminares del presente estudio, donde se concentra información sobre la importancia y el impacto percibido del turismo por las comunidades investigadas, la valoración de los resultados, los apoyos gubernamentales recibidos y la iniciativa para la creación de las unidades de negocios.

\subsection{Resultados por comunidad}

\subsubsection{Manantiales de Zacualpan}

El cese de operaciones del balneario Manantiales de Zacualpan representa el caso más extremo de las amenazas que enfrenta una unidad de negocios de твс. El balneario fue una víctima colateral de un intenso conflicto por recursos que ocurrió en la comunidad indígena de Zacualpan, Comala, originado en el extranjero y en las altas esferas del gobierno 
CuAdRo 5. Impactos y apoyos de las unidades de negocios estudiadas

\begin{tabular}{|c|c|c|c|c|c|c|}
\hline Municipio & & & COMALA & & & COLIMA \\
\hline $\begin{array}{l}\text { Unidad de } \\
\text { negocios }\end{array}$ & $\begin{array}{c}\text { Centro } \\
\text { Recreativo } \\
\text { La María }\end{array}$ & $\begin{array}{c}\text { Centro } \\
\text { Turístico } \\
\text { Carrizalillo }\end{array}$ & $\begin{array}{c}\text { Parador } \\
\text { "Gastronómico" }\end{array}$ & $\begin{array}{c}\text { Parador } \\
\text { "Gastronómico" }\end{array}$ & $\begin{array}{l}\text { Manantiales } \\
\text { de Zacualpan }\end{array}$ & El Cahuite \\
\hline Comunidad & La Becerrera & Suchitlán & $\begin{array}{l}\text { Cofradía de } \\
\text { Suchitlán }\end{array}$ & La Nogalera & Zacualpan & Acatitán \\
\hline Población & 274 & 4083 & 1601 & 354 & 1724 & 191 \\
\hline Trabajadores & 7 & 4 & ND & 17 & ND & 14 \\
\hline $\begin{array}{l}\text { Importancia } \\
\text { de la } \\
\text { actividad }\end{array}$ & 1 & 3 & 3 & 1 & ND & 2 \\
\hline Resultados & Moderados & Limitados & Limitados & Muy positivos & Eliminados & $\begin{array}{l}\text { Muy } \\
\text { positivos }\end{array}$ \\
\hline Iniciativa & Gubernamental & Gubernamental & Imitación & Mujeres & Comunitaria & Comunitaria \\
\hline $\begin{array}{l}\text { Apoyos } \\
\text { oficiales }\end{array}$ & $\begin{array}{l}\text { Construcción } \\
\text { de } \\
\text { instalaciones }\end{array}$ & $\begin{array}{l}\text { Construcción } \\
\text { de } \\
\text { instalaciones }\end{array}$ & Nulos & Reducidos & $\begin{array}{l}\text { Construcción } \\
\text { de } \\
\text { instalaciones }\end{array}$ & $\begin{array}{l}\text { Construcción } \\
\text { de } \\
\text { instalaciones }\end{array}$ \\
\hline
\end{tabular}

Fuente: Elaboración propia.

federal, y en el cual intervino una gran cantidad de actores internos y externos a la comunidad. El desenlace fue la suspensión de operaciones y la destrucción de las instalaciones del balneario. Básicamente, Manantiales de Zacualpan ilustra el caso de una unidad de negocios de TBC gestionada sin transparencia y sin rendición de cuentas, con los dirigentes actuando a espaldas de la población y buscando su beneficio particular.

Zacualpan es la única localidad del estado de Colima con población indígena y propiedad comunal de la tierra y los recursos. El balneario comunitario venía operando con cierta regularidad hasta noviembre de 2013, cuando una empresa minera recibió una concesión del gobierno federal para explotar una superficie de 200 hectáreas, casi la totalidad de la superficie de la comunidad; el objetivo de la empresa minera era extraer oro, plata y cobre del territorio comunitario. Dentro de la comunidad surgió una profunda división, y la mayoría rechazó la presencia de la mina, lo cual llevó a la destitución del presidente del Comisariado de Bienes Comunales de Zacualpan, quien era apoyado por un grupo minoritario.

En noviembre de 2013 se creó la organización denominada Consejo Indígena por la Defensa del Territorio de Zacualpan, con el propósito expreso de impedir la instalación de la empresa minera en la comunidad. El gobierno del estado de Colima, con la intervención de la Procuraduría Agraria, ha intentado convertir el régimen de propiedad comunal de la localidad en régimen ejidal, para que posteriormente pueda devenir propiedad privada y desparezca el régimen comunal (Red Mexicana de Afectados por la Minería, 2017). 
Además, la operación de la mina contaminaría el manantial de Zacualpan, del cual se abastecen las ciudades de la zona conurbada de la capital estatal, integrada por los municipios de Colima y Villa de Álvarez, con una población cercana a los 290000 habitantes.

En el conflicto han intervenido el gobierno federal, el gobierno de Colima, el ayuntamiento de Comala, el congreso del estado y el Tribunal Unitario Agrario local. Apoyando a los comuneros que rechazan la instalación de la mina, participaron la organización ecologista Biosiguana A. C., el Frente de Pueblos Indígenas de Puebla, Tlaxcala, Morelos y la Red Mexicana de Afectados por la Minería. El caso de Zacualpan se registra entre los 265 conflictos entre comunidades y empresas mineras documentados por el Observatorio de Conflictos Mineros de América Latina (2018). La división de la comunidad ha ocasionado episodios de violencia, y hasta este momento el enfrentamiento entre sus integrantes impide la reapertura del balneario, cuyas instalaciones fueron destruidas en actos de vandalismo promovidos por miembros del grupo minoritario de la comunidad aliado con la empresa minera.

\subsubsection{Centro Turístico Carrizalillos}

Fundado en 1973 por iniciativa gubernamental en el ejido Suchitlán, le fueron donadas todas las instalaciones de que dispone, a saber, habitaciones hoteleras, restaurante (en la actualidad concesionado), canchas, cobertizos, casetas y caminos. Hoy en día ofrece servicios complementarios como alquiler de lanchas, bicicletas y caballos. Su administración depende del ejido, y su operación se ha visto interrumpida ocasionalmente. Entre sus principales fortalezas se encuentran sus vistas a los volcanes y su ambiente apacible. Si bien cuenta con habitaciones hoteleras, su principal fuente de ingresos son grupos de paseantes que emplean los cobertizos para asar carne o hacer pícnics. Está posicionado como un lugar de paseo entre la población regional y los visitantes, aunque el volumen de su operación turística es exigua.

Genera cuatro empleos y difícilmente puede considerarse un éxito comercial o financiero. El impacto socioeconómico de sus actividades es percibido por la comunidad como muy limitado; sus instalaciones lucen abandonadas. El problema primordial que enfrenta la unidad de negocios es la amenaza de la desaparición de la laguna, su principal atractivo, la cual disminuyó de manera alarmante su nivel durante el estío del año 2017. Existen diversas explicaciones tentativas al riesgo de desaparición del cuerpo de agua; entre otras, que existen grietas en su fondo que filtran el agua; se han mencionado la deforestación, el efecto barrera de la carretera que la circunda, la escasez de lluvias y el desvío de sus fuentes para fines agrícolas. Finalmente, el deterioro de las condiciones de la laguna se atribuye a problemas de gestión y a sus limitados ingresos, que impiden brindarle el mantenimiento apropiado.

Desde el punto de vista del TBC, no se cumplen los planteamientos de la sustentabilidad ambiental, pues los encargados de la gestión de la unidad de negocios no muestran el interés ni la capacidad requerida para aprovechar, mantener y conservar sus atractivos y recursos turísticos. 


\subsection{3 "Paradores gastronómicos" de La Nogalera y Cofradía de Suchitlán}

Los paradores gastronómicos de La Nogalera y Cofradía de Suchitlán agrupan un conjunto de restaurantes, cafeterías y fondas que se han establecido a la orilla de la carretera Comala-San Antonio, sobre la zona federal, aprovechando el tránsito de viajeros, turistas y paseantes que se genera sobre la carretera federal 16 de Colima; en su mayoría, son establecimientos irregulares y espontáneos que toman la oportunidad económica ofrecida por el tránsito de pasajeros. Es un caso típico del efecto imitación, pues la actividad fue iniciada en la comunidad de La Nogalera por una señora procedente del Estado de México con experiencia en la operación de establecimientos de alimentos y bebidas, originando la atracción de comensales; las mujeres de la comunidad, al percatarse de la afluencia de clientela, decidieron abrir sus propios negocios. Con el paso del tiempo, la cantidad de estos creció, hasta integrar un "clúster" de fondas de comida típica que se ha popularizado entre visitantes y la población de los municipios aledaños. Las operadoras de los negocios han recibido algunos apoyos gubernamentales menores y concesiones provisionales restringidas para ejecutar sus actividades en la zona federal.

En rigor, estos conglomerados de establecimientos no pueden considerarse una unidad de negocios, como tampoco presentan una figura organizacional de TBC. De hecho, son un conjunto de establecimientos independientes y rústicos, desarrollados por las mujeres de la comunidad. El apelativo "parador gastronómico" que le ha sido asignado por las autoridades locales resulta claramente un eufemismo, pues los alimentos ofrecidos quedan en la categoría de comida casera o antojitos típicos, lo cual no afecta su éxito comercial. Sin embargo, las encuestas y entrevistas realizadas en la comunidad muestran que los establecimientos han tenido un importante impacto socioeconómico entre la población, en especial por ser una iniciativa de mujeres, que canalizan la totalidad de sus ingresos al hogar y a la familia.

El parador gastronómico de Cofradía de Suchitlán es un caso de efecto imitación de una imitación. Algunos emprendedores de la comunidad, al percibir el éxito comercial registrado por los negocios de La Nogalera, ubicándose sobre la misma vía y en la misma zona, decidieron organizarse y crear su propio corredor. Existen siete establecimientos de alimentos y bebidas, de los cuales cuatro son cocinas económicas, dos son cafeterías y uno es merendero. Al principio hubo una organización que se encargó de gestionar los permisos para situarse a la orilla de la carretera frente a la Secretaría de Comunicaciones y Transportes; por necesidades económicas, el líder de la incipiente organización emigró al vecino país del norte y esta se desintegró. No obstante, los establecimientos continúan operando, aunque los titulares de los permisos los transfirieron o subarrendaron. Sus características y su oferta de alimentos y bebidas son más heterogéneas que en La Nogalera y sus resultados económicos son mixtos: algunos logran mayores rendimientos que otros.

Por su falta de organización formal y su modalidad de gestión, los paradores gastronómicos de La Nogalera y Cofradía de Suchitlán no pueden considerarse unidades de negocios de TBC, a pesar de que en La Nogalera los empleos generados, el impacto socioeconómico en la comunidad y la percepción del turismo como fuente de bienestar resultan muy positivos. 


\subsubsection{Centro Ecoturístico La María}

Presenta muchas similitudes con Carrizalillo, aunque con mejores resultados. También se trata de un conjunto de cabañas construidas alrededor de una laguna; cuenta con albercas, restaurantes, cobertizos, alquiler de lanchas, canchas, espacio para acampar y pesca. El centro fue financiado y construido por el gobierno federal en la misma época en que se creo Carrizalillo; su gestión es ejidal y su operación se ha visto ocasionalmente interrumpida. El impacto de la actividad económica percibido en la comunidad es más palpable; su cuerpo de agua y sus instalaciones se encuentran en buen estado y la comunidad brinda gran importancia a la actividad turística; genera siete empleos. Sus efectos socioeconómicos pueden considerarse moderados.

\subsubsection{Centro Ecoturístico "El Cahuite"}

Ubicado en el municipio de Colima, este centro turístico es propiedad de ejidatarios, pero para su gestión se integraron en una Sociedad de Producción Rural. Inició operaciones en 2002; sus instalaciones han sido financiadas por diversos organismos gubernamentales, con una diferencia relevante: la iniciativa surgió de los propios ejidatarios. El atractivo principal del centro es un conjunto de albercas alimentadas con agua de manantial, que ha sido complementado con toboganes, canchas, cobertizos, tienda y restaurante; su mercado se integra por segmentos de clase media y popular, y registra una gran demanda. Genera 14 empleos e importantes ingresos, en comparación con el resto de las unidades de negocios estudiadas. Presenta un elevado nivel de organización y liderazgo competente; en mercadotecnia, cuenta con su propia página web y Facebook. Sus resultados socioeconómicos pueden considerarse muy positivos, sin embargo, existe cierto resentimiento de algunos integrantes de la comunidad que no participan en el negocio, básicamente porque decidieron no invertir al momento de comenzar el proyecto.

Puede decirse que, originalmente, el Centro Ecoturístico “El Cahuite” se concibió como un proyecto de TBC que, más tarde, a fin de incrementar su eficiencia y sus rendimientos, evolucionó hacia una forma de organización empresarial, aunque los beneficios de la empresa alcanzan a la mayoría de los ejidatarios y a la comunidad de Acatitán en general.

\subsection{Resultados generales de la encuesta a la población}

El cuestionario utilizado se adaptó del formato de Croes y Rivera (2015) e incluye dos variables: la primera mide la satisfacción con la vida y la segunda contempla la percepción del turismo como fuente de bienestar por la población de las comunidades estudiadas; los resultados de la primera variable fueron inconsistentes, por lo que no se incluyen en la presentación de resultados. La segunda variable se compone de 21 ítems formulados en la modalidad de escala de Likert de 5 puntos. Los resultados de los datos recabados mediante los 908 cuestionarios válidos obtenidos se procesaron con el software sPSs 22 , presentando una distribución normal y un alfa de Cronbach de 0.9 (figura 1). 


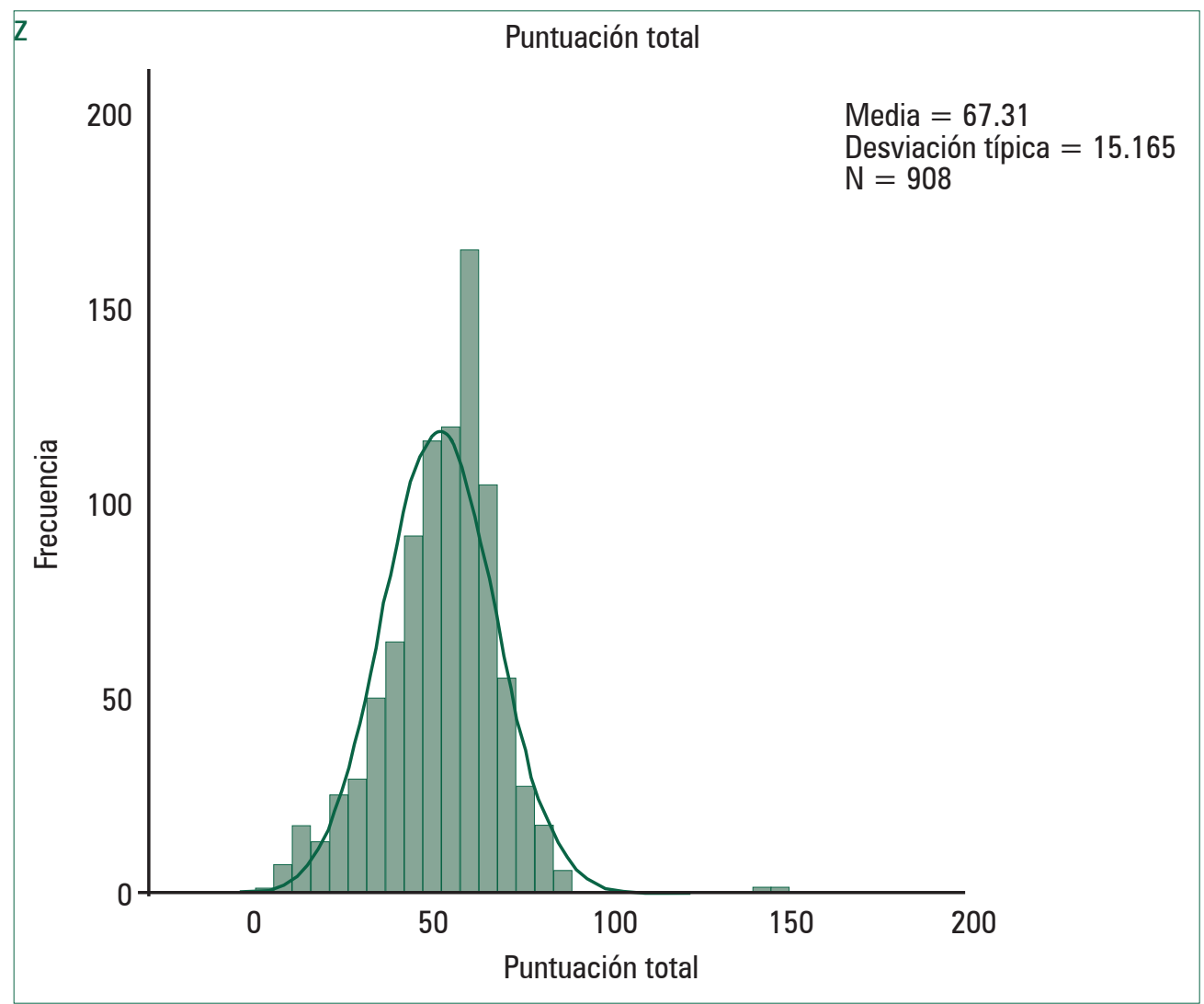

Fuente: Elaboración propia.

FIgURA 1. Distribución normal de los datos de la encuesta recabada en las comunidades estudiadas

La figura 2 muestra los resultados de la percepción general del turismo como fuente de bienestar en las comunidades examinadas, registrando un resultado promedio de 68.59 $\%$. Los resultados más bajos se obtienen en la comunidad de Zacualpan, donde los diversos conflictos llevaron a la desaparición de la unidad de negocios. Los resultados más positivos son para La Nogalera, donde las mujeres de la comunidad operan el "corredor gastronómico". Llama la atención que Acatitán aparece en tercer lugar, con la unidad de negocios más eficiente y productiva del conjunto, lo cual parece explicarse por la percepción de aquellos habitantes de la comunidad que no participan en el exitoso negocio turístico. Los resultados de Suchitlán y La Becerrera parecen derivar de los resultados económicos limitados registrados por las unidades de negocios de Carrizalillos y La María. 


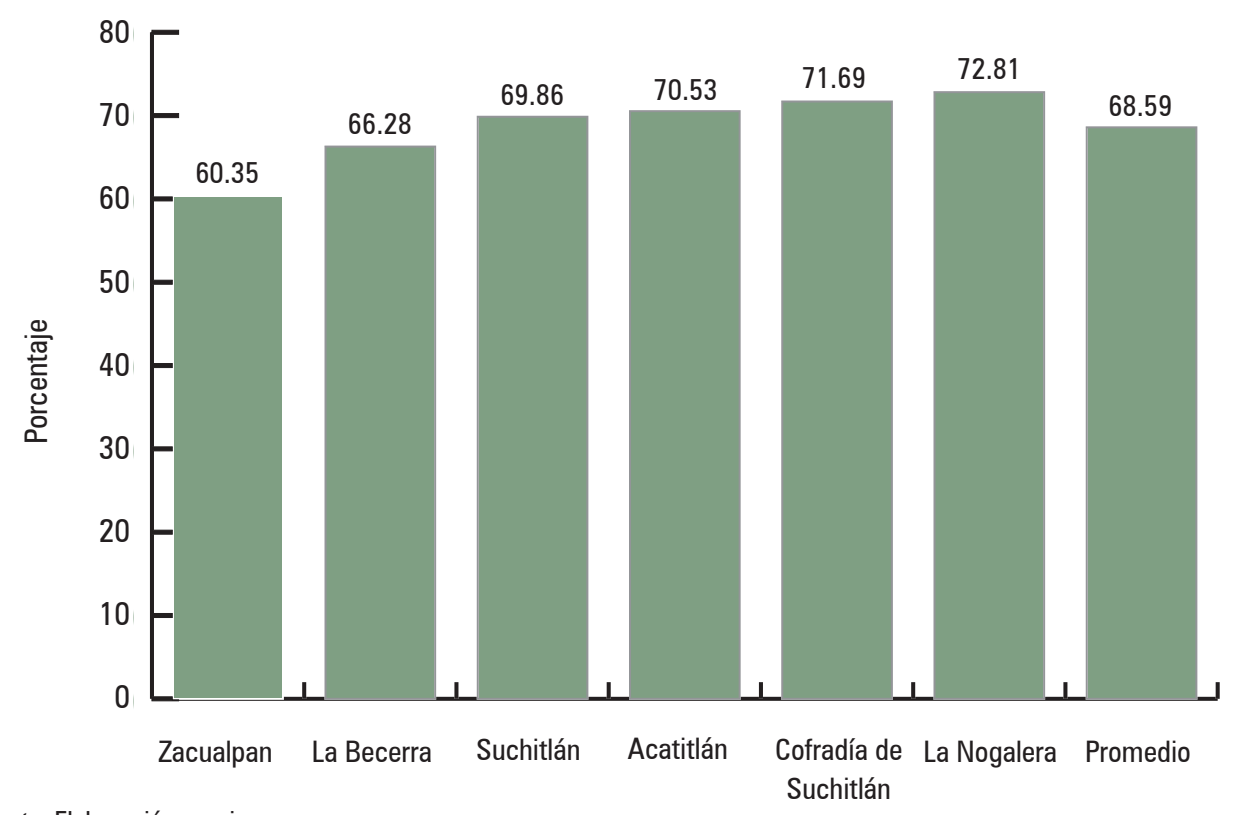

Fuente: Elaboración propia.

Figura 2. Percepción del turismo como fuente de bienestar en las comunidades rurales

\section{Discusión}

Con la información recabada y analizada hasta este momento en la investigación en curso, pueden extraerse algunas conclusiones preliminares. De entrada, más que operadores de TBC, parece tratarse de grupos de pobladores rurales dedicados a trabajos tradicionales en agricultura o ganadería en busca de ingresos complementarios, que de manera imprevista se ven convertidos en operadores turísticos, motivados por la oportunidad de generar recursos adicionales para sus familias. Uno de los factores que contribuyen a comprender la percepción del turismo como fuente de bienestar entre la población de las comunidades encuestadas es la denominación de Comala como pueblo mágico, que ha influido en la proliferación de empresas turísticas e impulsado la cultura turística; de hecho, las empresas más importantes del municipio son turísticas, dejando en segundo término a las actividades agropecuarias tradicionales.

Los resultados del trabajo sugieren que, más que referirse a TBC, es apropiado mencionar unidades de negocios turísticas ubicadas en el medio rural; en la muestra estudiada, dos de ellas fueron iniciativas gubernamentales completamente financiadas y equipadas por el gobierno federal: los centros turísticos de Carrizalillos y La Becerrera; el resto fueron iniciativas campesinas. Solo en dos de las unidades de negocios examinadas se puede hablar de un empoderamiento de la comunidad en los términos propuestos por Goodwin y Santilli (2009): Acatitán y La Nogalera; del resto, el parador gastronómico de Cofradía de 
Suchitlán y los centros turísticos de Carrizalillos y La María puede decirse que subsisten. El balneario de Zacualpan se autodestruyó por conflictos internos derivados de la falta de transparencia y rendición de cuentas en la administración de sus recursos frente a la comunidad, resultando una lamentable ilustración del incumplimiento de esos principios, de la manera en que proponen Dixey (2005), Asker et al. (2010), Silva y Wimalaratana (2013), Salazar (2012) y el Banco Mundial (The World Bank, 2013).

Como beneficiarios principales de las unidades de negocios estudiadas se encuentran los dirigentes, los trabajadores y sus familias, con la población de las comunidades beneficiándose de forma secundaria e indirecta. Goodwin y Santilli (2009) señalan atinadamente que, en algunos casos, la población de las comunidades es demasiado grande para que los ingresos generados por sus unidades de negocios turísticas logren alcanzar a todos los habitantes, como sucede con Zacualpan y Suchitlán.

Los resultados preliminares de la investigación sugieren que mientras mayor es el número de empleos creados por la unidad de negocios, en relación con el tamaño de la población de la comunidad, mayor es la percepción del turismo como fuente de bienestar por los habitantes, como puede observarse en el cuadro 5, en función de la cantidad obtenida de entrevistas a trabajadores. Croes (2014) y Croes y Rivera (2015) mencionan la capacidad del turismo para superar la pobreza, que a su vez parece derivarse en gran medida de la capacidad de la unidad de negocios para generar empleos.

Entre los beneficios del TBC indicados por Twining-Ward (2007), presentados en el cuadro 1, prevalece el económico sobre el sociocultural y el ambiental; entre las comunidades y unidades de negocios estudiadas no se logró identificar la determinación o algún interés especial por la protección del medio ambiente y el fortalecimiento de la identidad sociocultural.

La demanda por los servicios también aparece como un factor de éxito relevante, del modo en que propone el Banco Mundial (The World Bank, 2013); en el caso del presente estudio, la demanda por las albercas de El Cahuite y por los antojitos tradicionales de La Nogalera presenta un importante contraste frente a los servicios de hospedaje existentes en La María y Carrizalillos. Esto parece indicar que ofrecer servicios más sencillos resulta más conveniente que presentar un abanico más amplio de opciones; Goodwin y Santilli (2009) ya han señalado que la oferta de servicios de hospedaje no ha tenido éxito en los proyectos de turismo de base comunitaria, a pesar de que Responsible Travel (2013) incluye en su definición de твс a la provisión de hospedaje.

Autores como Salazar (2012), Kyrgyz Community Based Tourism Association (2013), Silva y Wimalaratana (2013) y Goodwin y Santilli (2009) han apuntado como principios fundamentales de la operación del turismo comunitario la rentabilidad y la viabilidad económica de las unidades de negocios, señalando numerosos casos de proyectos de esta modalidad que cierran operaciones al suspenderse los apoyos institucionales.

En relación con los proyectos estudiados, se observa que la participación de organismos gubernamentales de diversos niveles en el fomento al TBC ha sido inconstante; han distribuido importantes cantidades de recursos entre algunas de las unidades de negocios investigadas para construcción, habilitación y capacitación, entre otros rubros, solo que 
los recursos y apoyos se han entregado de manera intermitente, no se observa un seguimiento permanente, un acompañamiento continuo a las unidades de negocios.

Desde el punto de vista de los beneficiarios, el aprovechamiento y la gestión de los apoyos gubernamentales obtenidos parecen derivar de la iniciativa de los operadores, aunque los resultados no son concluyentes. Al analizar las dos unidades de negocios más exitosas se advierte que una de ellas prácticamente carece de estructura organizacional y del uso de herramientas de gestión (La Nogalera), mientras que El Cahuite cuenta con una sólida estructura organizacional que aplica herramientas de gestión y algunas tecnologías contemporáneas.

Los casos de los desastres sociales y ambientales de Zacualpan y Carrizalillos demuestran la fragilidad de las organizaciones comunitarias; en el primer caso, las poderosas fuerzas económicas y políticas de los intereses mineros internacionales pusieron en crisis a la comunidad, generando divisiones internas y enconos que hasta el momento no se han resuelto, sin que las autoridades hayan intervenido para lograr la reconciliación de los habitantes. En el segundo caso, se percibe desinterés, negligencia o incapacidad de los operadores turísticos para proteger las instalaciones y su recurso clave, la laguna. Estas dos unidades ilustran negativamente la falta de organización y de rendición de cuentas, la escasa participación de la población de las comunidades en la toma de decisiones y la ausencia de sustentabilidad en la operación turística.

En cuanto a los beneficios del TBC propuestos por Twining-Ward (2007), el desarrollo de infraestructura en algunas de las unidades de negocios estudiadas, como Carrizalillos y La María, no se ha visto reflejado en el incremento de empleos, ingresos y superación de la pobreza, aunque, en lo general, sí se ha generado cierta diversificación económica. En lo que se refiere al desarrollo sociocultural de la comunidad como producto de las actividades turísticas, parece presentarse solo en función de los resultados económicos, a la inversa de los planteamientos de ese autor. La sustentabilidad no se encuentra entre los asuntos prioritarios de las unidades de negocios examinadas, por lo menos no en los términos propuestos por Goodwin y Santilli (2009), Salazar (2012) y Kyrgyz Community Based Tourism Association (2013).

Si bien es cierto que la totalidad de las unidades de negocios estudiadas en la muestra ha recibido educación y capacitación por parte de organismos gubernamentales e instituciones de educación superior, en los términos planteados por Asker et al. (2010) y Goodwin y Santilli (2009), su impacto resulta limitado, al ser cursos o eventos de capacitación aislados, realizados en forma dispersa. En lo relativo a la gestión comercial y empresarial de la empresa como propone Dixey (2005), su presencia en las unidades de negocios analizadas resulta muy incipiente, con excepción de El Cahuite. De hecho, la gestión de las unidades de negocios más antiguas, como Carrizalillos y La María, ha exhibido una operación intermitente, con interrupciones recurrentes y cambios frecuentes de administración. 


\section{Conclusiones}

Los resultados preliminares del trabajo sugieren que los resultados económicos de la operación turística en las unidades de negocios estudiadas prevalecen sobre aspectos como la protección del medio ambiente, la identidad sociocultural y la organización de base comunitaria. Los volúmenes de ventas, las dimensiones de la demanda, la cantidad de empleos generados y el monto de los ingresos obtenidos parecen inducir una percepción del turismo como fuente de bienestar y una herramienta para superar la pobreza por la población de las comunidades. Aparentemente, estos indicadores tienen un peso mayor que la capacidad de organización y de gestión de los operadores turísticos, pues son factores comunes en los dos extremos de las unidades de negocios: La Nogalera, con las señoras cocineras operando sin organización formal ni herramientas de gestión, frente a los empresarios rurales de El Cahuite, que exhiben una sólida organización, técnicas de gestión profesionales y el empleo incipiente de tecnologías de información y comunicación.

En términos generales, los resultados económicos y la distribución de sus beneficios se manifiestan como el factor prevaleciente sobre un conjunto de elementos propuestos en la literatura académica para la gestión del TBC y su sustentabilidad. Los planteamientos en torno a la participación de la comunidad en la toma de decisiones, la gestión participativa, la sustentabilidad, el empleo de herramientas contemporáneas de gestión, el desarrollo de la comunidad, la sustentabilidad de la operación turística y la conservación de la identidad y la cultura local se presentan como asignaturas pendientes en la muestra investigada.

Los resultados del trabajo también apuntan a la iniciativa de los interesados como un factor potencial de éxito de las unidades de negocios analizadas; esto es, existen indicios de que aquellas unidades de negocios surgidas por iniciativa de los habitantes de la comunidad producen resultados más positivos que cuando la iniciativa del proyecto ha sido del gobierno. Esto puede ilustrarse comparando los casos de centros turísticos estancados, como Carrizalillo y La María, con el dinamismo de La Nogalera y El Cahuite, que pueden considerarse las unidades de negocios estudiadas en la muestra con mejores resultados económicos en términos de clientela y volúmenes de ventas. La iniciativa de los interesados como factor de éxito de las unidades de negocios turísticas en el medio rural no se menciona en la literatura académica consultada.

En relación con el tema de la demanda de servicios turísticos, un factor que pudiera inhibir el desarrollo de las unidades de negocios examinadas es la reducida captación de turistas nacionales y extranjeros en el estado de Colima; a pesar de la considerable cantidad de recursos naturales y culturales que posee, se ubica en el lugar 29 de 32 entidades federativas en términos de captación de turistas, de los cuales más de 95 \% son nacionales (Secretaría de Turismo, 2018); de hecho, la mayoría de la clientela de las unidades de negocios estudiadas es regional.

Otro componente que se ha mencionado como limitante del desarrollo de las unidades de negocios revisadas es la falta de diversificación de su oferta turística y su carácter 
pasivo; básicamente se trata de lagunas, manantiales y paisaje, con restringida oferta de actividades lúdicas, deportivas o de entretenimiento. Si bien existen los recursos, llama la atención la escasez de oferta de recorridos de aventura o de naturaleza, al igual que la ausencia de oferta turística basada en folclore, arte y tradiciones, propuestos por Tasci et al. (2013) como atractivos en el turismo de base comunitaria.

Así, puede destacarse que la operación turística habitual en las comunidades rurales dista mucho de corresponder con los planteamientos y elaboraciones teóricas de los académicos sobre el TBC, aunque la población de las comunidades examinadas sí percibe a la actividad turística como fuente de bienestar. Una vez que se concluya con el levantamiento y el análisis de la información, se presentará el reporte completo del estudio, realizando análisis en profundidad por cada una de las comunidades.

\section{Fuentes consultadas}

Asker, S., Boronyak, L., Carrard, R. y Paddon, M. (2010). Effective Community Based Tourism: A Best Practice Manual. Sustainable Tourism Cooperative Research Centre. Recuperado de http://publications.apec.org/-/media/APEC/Publications/2010/6/ Effective-Community-Based-Tourism-A-Best-Practice-Manual-June-2010/210_ twg_CommunityBasedTourismWEB.pdf

Blake, A., Saba, J., Sinclair, M. y Kuhl, V. (2008). Tourism and poverty relief. Annals of Tourism Research, 30(1), 107-126. Recuperado de https://www.sciencedirect.com/ science/article/abs/pii/S0160738307000916.

Coneval. (2018). Anexo único de los "Lineamientos y criterios generales para la definición, identificación y medición de la pobreza”. Actualización 2018. Metodología para la medición multidimensional de la pobreza en México. Recuperado de https://www. coneval.org.mx/Medicion/MP/Paginas/Lineamientos_DOF.aspx

Coneval. (2019). Medición de la pobreza. Evolución de las líneas de pobreza por ingresos. Recuperado de https://www.coneval.org.mx/Medicion/MP/Paginas/Lineas-de-bienestar-y-canasta-basica.aspx

Croes, R. (2014). Tourism and poverty reduction in Latin America: Where does the region stand? Worldwide Hospitality and Tourism Themes, 6(3), 293-300.

Croes, R. y Rivera, M. (2015). Poverty Alleviation through Tourism Development: A Comprehensive and Integrated Approach. Boca Ratón: CRC Press.

Dixey, L. (2005). Inventory and Analysis of Community Based Tourism in Zambia. Recuperado de http://fsg.afre.msu.edu/zambia/resources/PROFIT\%20Community\%20 Tourism\%20Survey\%20-\%20Final\%20CBT\%20Report.pdf

Donaldson, J. (2007). Tourism, development and poverty reduction in Guizhou and Yunnan. China Quarterly, 190, 333-351.

Fernández, M. (2011). Turismo comunitario y empresas de base comunitaria turísticas: ¿estamos hablando de lo mismo? El Periplo Sustentable, 20, 31-74.

Gascón, J. (enero, 2011). La metodología "Pro-Poor Tourism": un análisis crítico. Opiniones en Desarrollo. Programa Turismo Responsable. Artículo 9. Recuperado de://www. alainet.org/images/turismo.pdf 
Gobierno de la República. (2013). Plan Nacional de Desarrollo. Recuperado de http://pnd. gob.mx/

Goodwin, H. (2006). Measuring and Reporting the Impact of Tourism in Poverty. Recuperado de http://www.icrtourism.org

Goodwin, H. y Santilli, R. (2009). Community-Based Tourism: A Success? Recuperado de http://www.haroldgoodwin.info/uploads/cbtasuccesspubpdf.pdf

Gutiérrez-Pérez, F. J., Medina-Muñoz, D. R. y Medina-Muñoz, R. D. (2014). Turismo y alivio de la pobreza: una revisión de la literatura académica. Tourism \& Management Studies, 10(2), 104-115.

Hiwasaki, L. (2006). Community-based tourism: A pathway to sustainability for Japan's protected areas. Society and Natural Resources, 19(8): 675-692. doi: $10.1080 / 08941920600801090$

Hawkins, D. y Mann, S. (2007). The World Bank's role in tourism development. Annals of Tourism Research, 34(2), 348-363.

Instituto Nacional de Estadística y Geografía. (2018). Información por entidad. Recuperado de http://www.inegi.org.mx/

Jamieson, W., Goodwin, H. y Edmunds, C. (2004). Contribution of Tourism to Poverty Alleviation. Pro-Poor Tourism and the Challenge of Measuring Impacts. Recuperado de www.haroldgoodwin.info

Kyrgyz Community Based Tourism Association. (2013). Kyrgyz Community Based Tourism Association. Recuperado de http://www.cbtkyrgyzstan.kg/index.php/en/o-nas/ chto-takoe-tos

Manyara, G. y Jones, E. (2007). Community-based tourism enterprises development in Kenya: An exploration of their potential as Avenues of Poverty Reduction. Journal of Sustainable Tourism, XV(6), 628-644.

Mitchell, J. y Ashley, C. (2009). Tourism and Poverty Reduction. Pathways to Prosperity. Londres: Routledge.

Observatorio de Conflictos Mineros de América Latina. (2018). Conflicto minero: Proyecto minero en Zacualpán: saqueo y contaminación de agua. Recuperado de https:// mapa.conflictosmineros.net/ocmal_db-v2/conflicto/view/906

Red Mexicana de Afectados por la Minería. (2017). Zacualpan y su lucha contra la minería. Recuperado de http://www.remamx.org/category/colima/

Responsible Travel. (2013). Responsible Travel. Recuperado de http://www.responsibletravel.com/copy/what-is-community-based-tourism

Salazar, N. (2012) Community-based cultural tourism: Issues, threats and opportunities. Journal of Sustainable Tourist, 20(1), 9-22.

Scheyvens, R. y Russell, M. (2012). Tourism, land tenure and poverty alleviation in Fiji. Tourism Geographies: An International Journal of Tourism Space, Place and Environment, 14(1), 1-25.

Secretaría de Turismo. (2018). DATATUR. Compendio Estadístico del Turismo en México 2017. Recuperado de https://www.datatur.sectur.gob.mx/SitePages/CompendioEstadistico.aspx

Silva, D. A. C. y Wimalaratana, W. (2013). Community Based Sustainable Tourism Development in Sri Lanka: Special Reference to Moneragala District. Recuperado de http:/ / www.haroldgoodwin.info/PPT/PPTMonaragala.pdf 
Tasci, A., Semrad, K. y Yilmaz, S. (2013). Community Based Tourism Finding the Equilibrium in the COMCEC Context Setting the Pathway for the Future. Ankara: COMCEC.

Thailand Community Based Tourism Institute. (2013). Thailand Community Based Tourism Institute. Recuperado de www.cbt-i.org

Torres, R. y Momsen, J. (2004). Challenges and potential for linking tourism and agriculture to achieve pro-poor tourism objectives. Progress in Development Studies, 4(4), 294-318.

Twining-Ward, L. (2007). A Toolkit for Monitoring and Managing Community-based Tourism. University of Hawaii, School of Travel Industry Management.

The World Bank. (2013). Regional Study on Community Based Tourism in the Caucasus. Recuperado de http://Inweb90.worldbank.org/eca/eca.nsf/d1e666886eb626e2852567d 100165168/780a475beed61c07852568fc005df707 / \$FILE/Regional\%20Studies\%20on\%20Community-Based\%20Tourism\%20in\%20the\%20Caucasus.pdf.

The World Bank. (2014). Policy Research Report 2014: A Measured Approach to Ending Poverty and Boosting Shared Prosperity: Concepts, Data, and the Twin Goals.

The World Bank. (2018). Poverty and Shared Prosperity 2018: Piecing Together the Poverty Puzzle. Washington, D. C.: Autor. Recuperado de http://www.worldbank.org/en/ research/publication/a-measured-approach-to-ending-poverty-and-boosting-shared-prosperity.

World Tourism Organization. (2004). Manual on Tourism and Poverty Alleviation - Practical Steps for Destinations. Madrid: Autor.

World Tourism Organization. (2010). Tourism and Poverty Alleviation. Recommendations for Action. Madrid: Autor.

Zapata, M., Hall, C., Lindo, P. y Vanderschaeghe, M. (2011). Can community-based tourism contribute to development and poverty alleviation? Lessons from Nicaragua. $\mathrm{Cu}$ rrent Issues in Tourism, 14(8), 725-749. 